\title{
NOUVELLE
}

\section{Organisation spatio- moléculaire des neurones D2R du striatum dévoilée par le séquençage d'ARN à haut débit}

Emma Puighermanal ${ }^{1}$, Laia Castell ${ }^{1}$, Jean-Antoine Girault ${ }^{2}$, Emmanuel Valjent ${ }^{1}$
${ }^{1}$ Université de Montpellier, Institut de génomique fonctionnelle (IGF), CNRS, Inserm, 141 rue de la Cardonille, 34094 Montpellier, France.

${ }^{2}$ Institut du Fer à Moulin, Inserm UMRS 1270, Sorbonne Université, Faculté des Sciences, 75005 Paris, France. emmanuel.valjent@igf.cnrs.fr
> Le striatum dorsal (dorsal striatum, DS) et son extension ventrale, le noyau accumbens (nucleus accumbens, NAc) sont des structures cérébrales sous-corticales impliquées dans la planification motrice, la prise de décision, la motivation et les apprentissages contrôlés par la récompense [1]. Des dysfonctionnements de ces structures sont associés à de nombreuses maladies neurologiques ou psychiatriques, notamment la maladie de Parkinson, le syndrome de Gilles de la Tourette, les troubles obsessionnels compulsifs et du spectre de l'autisme, ainsi que les comportements addictifs [2].

Le striatum est composé de neurones de projection GABAergiques appelés neurones épineux de taille moyenne (medium-sized spiny neurons, MSN), qui représentent environ $95 \%$ des neurones du striatum chez les rongeurs [1]. Les $5 \%$ restant sont constitués d'interneurones cholinergiques et d'une grande variété d'interneurones GABAergiques [3]. Les MSN, sur lesquels convergent des projections glutamatergiques de l'ensemble du cortex cérébral, du thalamus et des régions limbiques telles que l'hippocampe ou l'amygdale, intègrent et traitent les informations sensorielles ou contextuelles. Une fois sélectionnées, les informations pertinentes sont à leur tour relayées vers les structures de sortie des noyaux gris centraux, permettant ainsi l'exécution de réponses comportementales adéquates [1]. L'intégration de ces informations est modulée par les neurones dopaminergiques ascendants provenant de la substance noire (pars compacta) et de l'aire tegmentale ventrale. Ainsi, en attribuant une valeur motivationnelle positive ou aversive à ces informations sensorielles ou contextuelles, la dopamine permet une optimisation des réponses comportementales [4].

La dopamine libérée dans le striatum agit en activant des récepteurs de la dopamine de types D1 (D1R) et D2 (D2R), dont l'expression par les différents types de neurones striataux varie. Ces deux sous-types de récepteurs sont exprimés par les MSN, et après quelques décennies de controverses, il est maintenant admis que les MSN contiennent soit les DIR soit les $D 2 R$, et que seuls quelques-uns expriment les deux récepteurs dans le DS et le NAc [5]. L'expression des D2R n'est cependant pas limitée aux MSN puisque ces récepteurs sont également présents sur les interneurones cholinergiques [6]. Alors que de nombreuses études s'accordent sur le fait que les neurones du DS et du NAc exprimant les D2R participent conjointement mais différemment au contrôle moteur, à la prise de décision ou encore à la motivation, les raisons de cette diversité fonctionnelle restent énigmatiques. Récemment, nous avons mis en évidence l'existence d'une hétérogénéité moléculaire et fonctionnelle des neurones D2R le long de l'axe dorsoventral du striatum.

L'utilisation de la méthode RiboTag [7], qui permet d'identifier l'ensemble des ARN messagers (ARNm) associés aux ribosomes dans des populations cellu- laires et des circuits neuronaux identifiés, nous a permis de déterminer si les neurones D2R du DS et du NAc avaient des caractéristiques moléculaires distinctes. Pour tester cette hypothèse, nous avons produit la lignée de souris D2-RiboTag [8], dans laquelle la protéine ribosomique Rpl22 étiquetée avec I'hémagglutinine ne s'exprime que dans les MSN exprimant les D2R (D2-MSN) et dans les interneurones cholinergiques (Figure 1A). Après dissection, les ARNm présents dans les neurones D2R du DS et du NAc ont été isolés par immunoprécipitation des polysomes à l'aide d'un anticorps anti-hémagglutinine, puis identifiés par séquençage à haut débit (Figure $1 B, C)$. Une analyse bioinformatique a révélé que, parmi les 14454 transcrits séquencés codant des protéines, près de 3000 étaient plus abondants dans les neurones D2R du NAc et presque 4 000 dans ceux du DS, suggérant l'existence d'une hétérogénéité moléculaire insoupçonnée au sein des D2-MSN et des interneurones cholinergiques [9].

Les analyses «Gene ontology » ont révélé une dichotomie entre les gènes préférentiellement exprimés dans le DS ou dans le NAc, qui pourrait sous-tendre la diversité des rôles et des composants cellulaires et moléculaires des neurones D2R. À titre d'exemple, de nombreux gènes associés aux fonctions mitochondriales, notamment en relation avec le fonctionnement de la chaîne respiratoire, sont fortement exprimés dans les D2-MSN du DS. Ainsi, 26 des 44 gènes codant les protéines du complexe de la chaîne respiratoire I 
A Souris D2-RiboTag

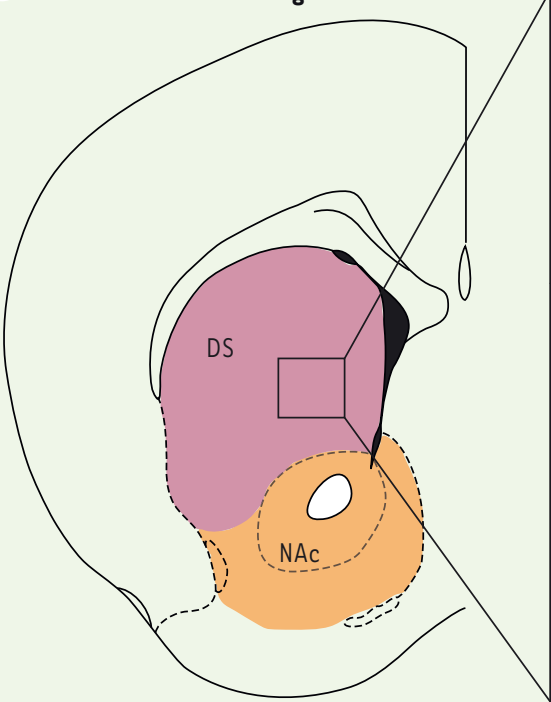

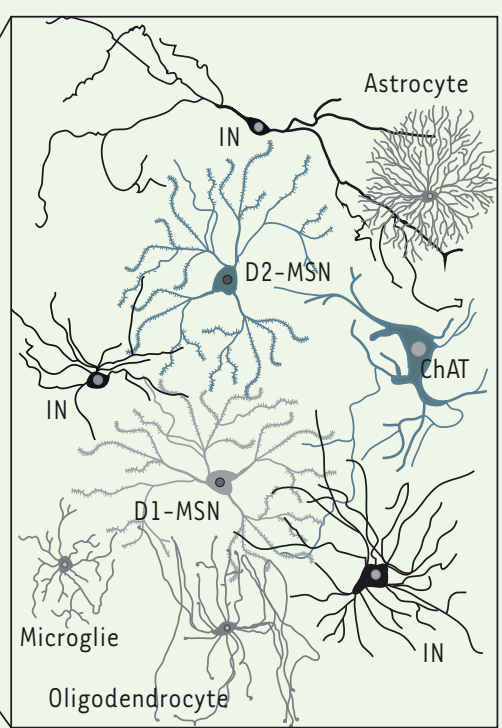

B

Homogénéisation du DS et du NAc

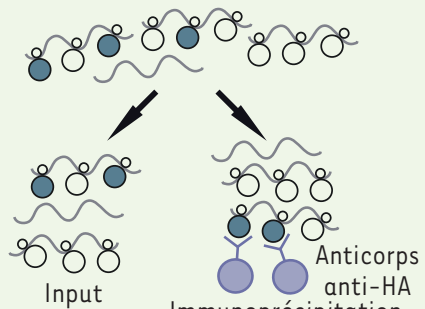

(10\% de

I'homogénat) $\varepsilon$

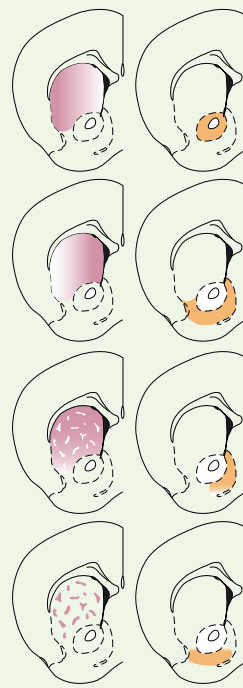

$F$

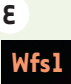

Wfsl
DS
Souris D2-eGFP
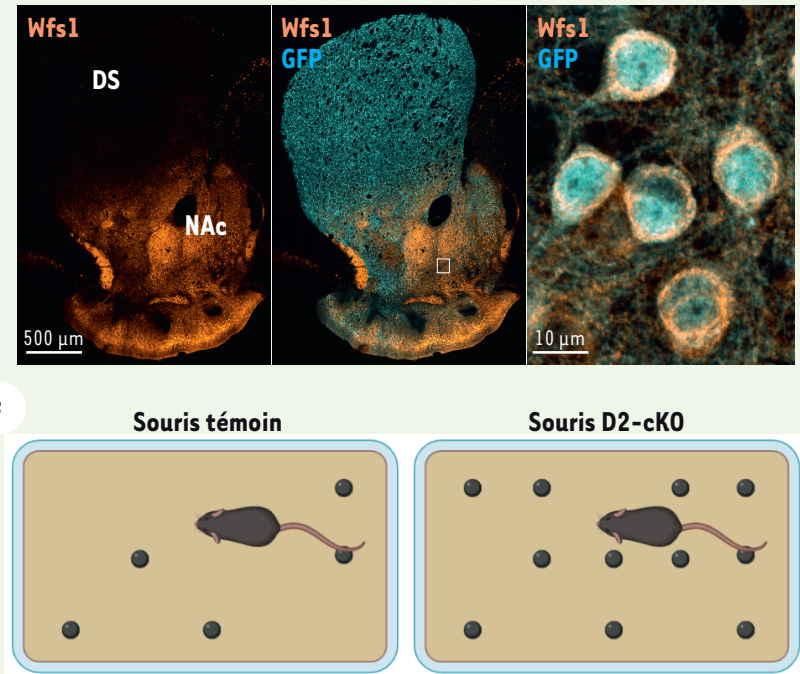

Immunoprécipitation

des polysomes

étiquetés HA

Purification des ARNm

Séquençage à haut débit des ARNm
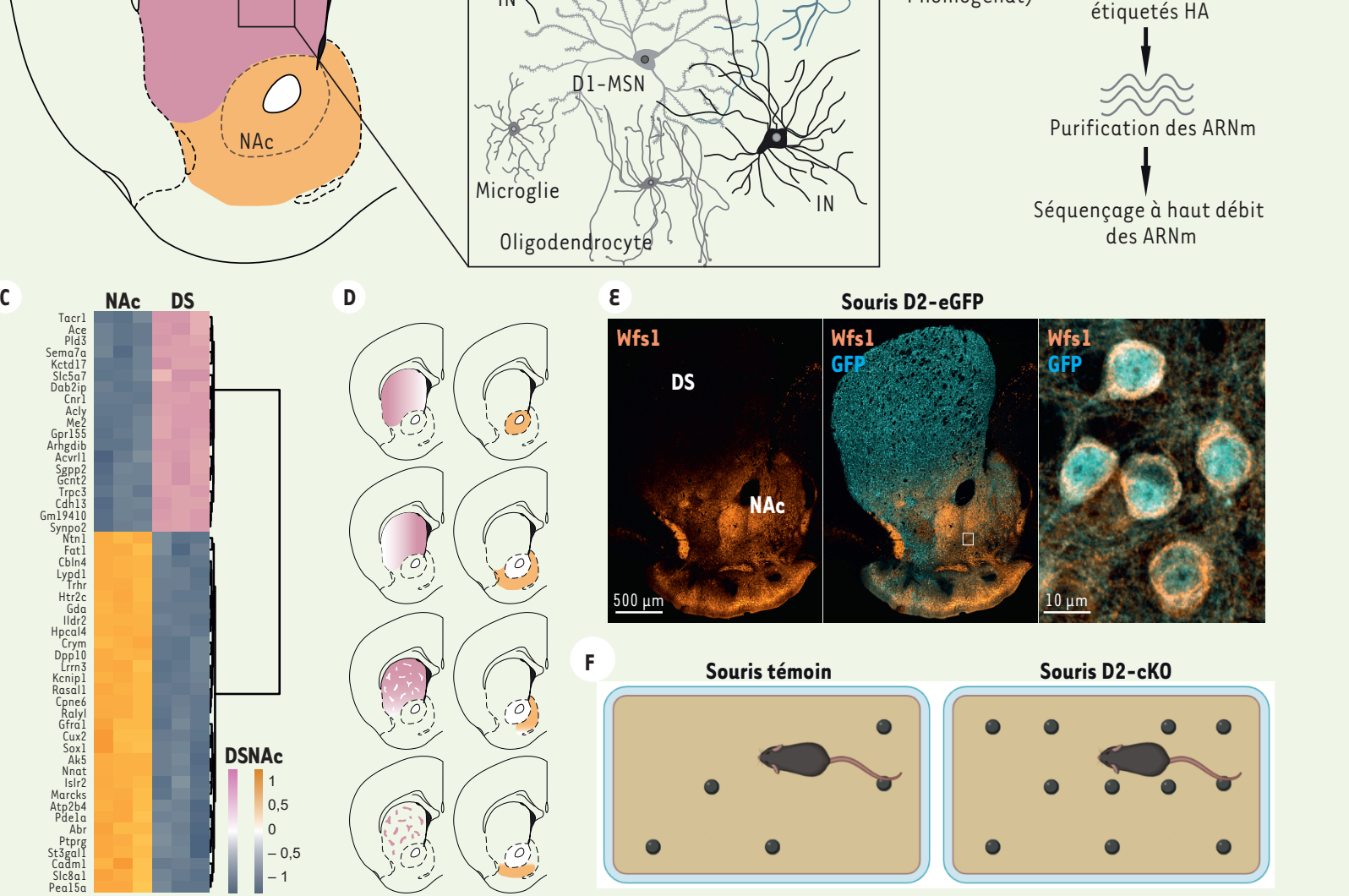

Figure 1. Hétérogénéité moléculaire et fonctionnelle des neurones D2R le long de l'axe dorso-ventral du striatum. A. Dessin d'une coupe coronale de cerveau de souris, où le striatum dorsal (DS) est délimité en rose et le noyau accumbens (NAc) en orange. Encadré schématisant les principaux types cellulaires présents dans le striatum. Les cellules gliales (astrocytes, oligodendrocytes) et microgliales sont représentées en gris foncé. Les neurones épineux de taille moyenne (medium-sized spiny neurons, MSN) exprimant le récepteur Dl de la dopamine (Dl-MSN) et les différents sous-types d'interneurones GABAergiques (IN) sont représentés respectivement en gris clair et en noir. Les D2-MSN et les interneurones cholinergiques (ChAT) exprimant la protéine ribosomique Rpl22 étiquetée avec l'hémagglutinine (HA) chez la souris D2-RiboTag sont représentés en vert-gris. B. Schéma descriptif de la méthode Ribotag. Après dissection et homogénéisation des échantillons tissulaires du DS et du NAc, les polysomes étiquetés avec l'hémagglutinine (HA) ont été immunoprécipités à l'aide d'un anticorps anti-HA. Une fois isolés, les ARNm présents dans les neurones du DS et du NAc exprimant le récepteur D2 de la dopamine ont été identifiés par séquençage à haut débit. C. Carte thermique (Heatmap) des 50 gènes les plus exprimés préférentiellement dans le DS (rose) ou dans le NAc (orange). Les niveaux d'expression sont codés par l'intensité des couleurs. D. Organisation spatio-moléculaire de neurones du DS et du NAc exprimant le récepteur D2 de la dopamine. Les gènes plus exprimés dans le DS ou dans le NAc ont des distributions spatiales hétérogènes et sélectives dans le DS médian, le DS latéral, les striosomes et la matrice du DS, ou encore dans le cœur du NAc et dans de nombreux sous-domaines de l'écorce du NAc. $\varepsilon$. Distribution spécifique de la protéine Wfsl dans le NAc (images de gauche et du milieu). L'image de droite est un agrandissement de la zone encadrée montrant l'expression de la protéine Wfsl dans les neurones du NAc exprimant le récepteur D2 de la dopamine, identifiés par la protéine fluorescente verte (GFP) chez des souris D2-eGFP. F. L'action de creuser est altérée chez les souris dans lesquelles les récepteurs D2 de la dopamine ont été invalidés sélectivement, à l'âge adulte, dans les neurones exprimant le gène WfsI (D2-cKO). Dans le test d'enfouissement des billes, le score d'enfouissement est diminué chez les souris D2-cKO. 
sont préférentiellement exprimés dans les neurones D2R du DS. Ce biais d'expression a des conséquences fonctionnelles puisque l'activité du complexe I de la chaîne respiratoire est plus forte dans le DS que dans le NAc [9]. Cette différence d'activité mitochondriale pourrait rendre compte de la vulnérabilité des D2-MSN du DS au stress oxydant. Ces observations sont particulièrement pertinentes dans le contexte de la maladie de Huntington puisque les D2-MSN du DS sont les premiers à dégénérer dans cette maladie.

Notre analyse a également permis de révéler l'expression préférentielle de nombreux gènes soumis à l'empreinte parentale ${ }^{1}$ dans les neurones D2R du NAc. Parmi les 90 gènes soumis à l'empreinte parentale détectés dans le striatum, plus de la moitié présentent une expression différentielle, 42 gènes étant plus exprimés dans le NAc et 19 dans le DS [9]. Nous avons notamment pu révéler la présence dans les neurones D2R du NAc de transcrits codant la tyrosine hydroxylase, l'enzyme limitante de la synthèse de la dopamine, alors même que la dopamine n'est pas produite dans le striatum [9]. Bien que la présence de la tyrosine hydroxylase dans ces neurones reste énigmatique, elle a cependant permis de définir une nouvelle sous-population de D2-MSN, dont la connectivité et le rôle restent à déterminer.

L'analyse des profils d'expression des gènes préférentiellement exprimés dans les neurones D2R du DS ou du

\footnotetext{
${ }^{1}$ Ce phénomène épigénétique, qui concernerait moins d $1 \%$ des gènes, conduit à l'expression par la cellule d'un seul des deux allèles parentaux : soit de l'allèle paternel, soit de
} l'allèle maternel, selon le gène.
NAc a révélé une distribution spatiale hétérogène qui suggère l'existence d'un code spatio-moléculaire (Figure ID) [9]. Ainsi, l'identification de centaines de nouveaux marqueurs moléculaires spécifiques de sous-domaines du DS et du NAc constitue une ressource de grande valeur pour cibler des souspopulations particulières de neurones $D 2 R$ et étudier leur rôle au sein de circuits striataux spécifiques [9]. Comme preuve de concept, nous avons caractérisé l'identité moléculaire d'une souspopulation neuronale du NAc définie par l'expression du gène $W f s l$ et déterminé l'impact fonctionnel de la délétion des D2R dans ces neurones (Figure $1 \varepsilon$ ). La création d'une lignée de souris dans laquelle les D2R ont été invalidés sélectivement, à l'âge adulte, dans les neurones exprimant $W f s I$ (D2-cKO) nous a permis de mettre en évidence le rôle joué par la dopamine dans l'action de creuser (Figure IF) [9]. Ainsi, ce trait comportemental conservé à travers l'évolution et utilisé par de nombreuses espèces pour chercher ou stocker de la nourriture, se protéger du froid ou des prédateurs, repose en partie sur la présence des D2R dans une sous-population de neurones du NAc définie par l'expression de Wfsl.

Par une approche de séquençage à haut débit des ARNm exprimés par les neurones D2R du DS et du NAc suivi d'une analyse bioinformatique, nous avons pu mettre en évidence l'existence d'une hétérogénéité moléculaire et fonctionnelle des neurones D2R de ces deux structures. Des analyses multiparamétriques croisées ont également permis de mettre en lumière l'existence d'une organisation spatio-moléculaire des neurones D2R du striatum et d'identifier une sous-population de neurones du NAc dans lesquels les D2R jouent un rôle spécifique. Ces résultats illustrent l'intérêt de cibler des sous-populations spécifiques de neurones du DS et du NAc afin de mieux appréhender les effets de la dopamine sur ses neurones cibles. $\diamond$ Molecular heterogeneity of striatal D2 neurons

\section{LIENS D'INTÉRÊT}

Les auteurs déclarent n'avoir aucun lien d'intérêt concernant les données publiées dans cet article.

\section{RÉFÉRENCES}

1. Gerfen CR, Surmeier DJ. Modulation of striatal projection systems by dopamine. Annu Rev Neurosci 2011 ; 34 : 441-66.

2. Maia TV, Frank MJ. From reinforcement learning models to psychiatric and neurological disorders. Nat Neurosci $2011 ; 14$ : 154-62.

3. Tepper JM, Koós T, Ibanez-Sandoval 0, et al. Heterogeneity and diversity of striatal GABAergic interneurons: update 2018. Front Neuroanat 2018; $124: 91$

4. Bromberg-Martin ES, Matsumoto M, Hikosaka 0. Dopamine in motivational control: rewarding, aversive, and alerting. Neuron $2010 ; 68: 815-34$

5. Valjent $\varepsilon$, Bertran-Gonzalez J, Herve D, et al. Looking $B A C$ at striatal signaling: cell-specific analysis in new transgenic mice. Trends Neurosci 2009 ; 32 : 538-47.

6. Matamales M, Bertran-Gonzalez J, Salomon L, et al. Striatal medium-sized spiny neurons: identification by nuclear staining and study of neuronal subpopulations in BAC transgenic mice. PLoS One 2009; 4 : e4770.

7. Sanz $\varepsilon$, Yang L, Su T, et al. Cell-type-specific isolation of ribosome-associated mRNA from complex tissues. Proc Natl Acad Sci USA 2009 ; 106 : 13939-44.

8. Puighermanal $\varepsilon$, Biever A, Espallergues J, et al. Drd2cre:ribotag mouse line unravels the possible diversity of dopamine D2 receptor-expressing cells of the dorsal mouse hippocampus. Hippocampus $2015 ; 25$ : 858-75.

9. Puighermanal $\varepsilon$, Castell L, Esteve-Codina A, et al. Functional and molecular heterogeneity of D2R neurons along dorsal ventral axis in the striatum. Nat Commun $2020 ; 11: 1957$.

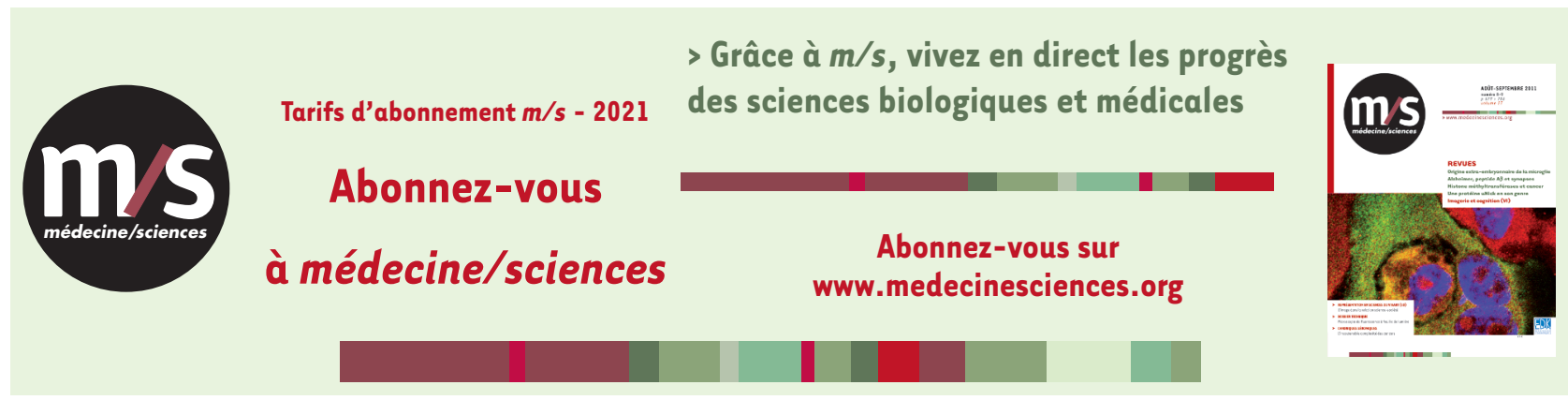

